\title{
Estrategia de eficiencia energética para redes WDM dinámicas
}

\author{
Energy efficiency strategy for dynamic WDM networks
}
A. Leiva ${ }^{1}$
I. Peralta ${ }^{1}$
F. Alonso ${ }^{1}$
H. Vargas ${ }^{1}$
S. Fingerhuth ${ }^{1}$

Recibido 4 de julio de 2013, aceptado 20 de mayo de 2014

Received: July 4, 2013 Accepted: May 20, 2014

\begin{abstract}
RESUMEN
Se propone y evalúa un mecanismo de funcionamiento de redes WDM (Wavelength Division Multiplexing) dinámicas con la finalidad de reducir el consumo energético de la capa óptica de estas sin afectar el rendimiento en términos de probabilidad de bloqueo de las conexiones.

El mecanismo propuesto, denominado Load-Aware Turn On-Off, consiste en controlar continuamente (a cada hora) el número de transpondedores optoelectrónicos (transmisores/receptores WDM y regeneradores) encendidos y/o apagados de los nodos WDM de acuerdo con la carga de tráfico ofrecida a la red y a la probabilidad de bloqueo máxima permitida. Resultados obtenidos mediante simulación, considerando la topología física de la NSFNet (National Science Foundation's Network), demuestran que este método de funcionamiento puede ahorrar $15 \%$ de consumo de energía comparado con una red WDM dinámica que no cuenta con estrategias de eficiencia energética.

Estos resultados pueden ser de interés a los operadores de telecomunicaciones que se encuentren evaluando la factibilidad de implementar redes WDM dinámicas en un futuro.
\end{abstract}

Palabras clave: Consumo energético, capa óptica, redes WDM dinámicas, carga de tráfico, probabilidad de bloqueo.

\begin{abstract}
This article proposes and evaluates a novel mechanism for operating dynamic WDM (Wavelength Division Multiplexing) networks, seeking to reduce the energy consumption in the optical layer of the network components, without affecting the network performance in terms of the blocking probability of connections. This mechanism, called Load-Aware Turn On-Off, consists in continuously (hourly) controlling the number of optoelectronic transponders (WDM transmitters/receivers and regenerators) turned on and/or turned off in WDM nodes, depending on changes in both the network traffic load and the maximum blocking probability allowed. The proposal has been tested with the NSFNet (National Science Foundation's Network) and results show that the method can save 15\% of the energy consumption regarding the operation of the same NFSNet without the energy efficiency strategy.

Simulation results presented in this paper could be of interest to telcos who are currently evaluating the feasibility to implement dynamic WDM networks.
\end{abstract}

Keywords: Energy consumption, optical layer, dynamic WDM networks, traffic load, blocking probability.

\section{INTRODUCCIÓN}

Las tecnologías de telecomunicaciones contribuyen con $10 \%$ del consumo energético mundial [1]. Sin embargo, se espera que este porcentaje aumente en los próximos años debido al gran crecimiento de tráfico de datos de las redes de telecomunicaciones (35 al 50\% anual [2]), lo que provocaría el uso

\footnotetext{
1 Escuela de Ingeniería Eléctrica. Pontificia Universidad Católica de Valparaíso. Av. Brasil 2147. Valparaíso, Chile. E-mail: ariel.leiva@ucv.cl; iperalta@grupogtd.com; alonsofrancisco@gmail.com; hector.vargas@ucv.cl; sebastian.fingerhuth@ucv.cl
} 
de dispositivos de mayor capacidad y consumo energético. Por esta razón es que en el último tiempo se han generado diversas propuestas sobre técnicas de eficiencia energéticas aplicadas a redes de telecomunicaciones (Green Networks [3-5]).

Los diseñadores de la capa óptica WDM de las redes de transporte también se han sumado a los esfuerzos de eficiencia energética. La capa óptica WDM de estas redes consiste en un conjunto de nodos de red (en este caso, nodos ópticos) que se encuentran conectados entre sí por medio de fibras ópticas por las que se propaga información de diversas fuentes de manera simultánea utilizando para ello portadoras ópticas (longitudes de onda) distintas. Experimentalmente se han implementado enlaces de hasta 224 longitudes de onda, cada una operando a $548 \mathrm{Gbps}$, obteniendo una capacidad total de 102,3 Tbps [6] por fibra óptica.

Actualmente las redes ópticas WDM operan de manera cuasiestática, es decir, las conexiones en la capa óptica son del tipo permanente con escalas de tiempo de meses a años [7]. Sin embargo, debido a la capacidad de adaptación a los cambios de tráfico y topologías, en los últimos años se han elaborado diversas propuestas de redes WDM del tipo dinámicas, como por ejemplo: redes OPS (Optical Packet Switching) [8], OBS (Optical Burst Switching) [9], WR-OBS (Wavelength-Routed Optical Burst Switching) [10], OCS (Optical Circuit Switching) [11], donde las conexiones ópticas se establecen bajo demanda y la duración de estas varía de fracciones de segundos a horas o días.

Las ventajas de la operación dinámica de la capa óptica WDM han provocado que especialistas en el área den por hecho la futura migración de las actuales redes WDM estáticas a redes WDM dinámicas [12-14]. Sin embargo, estudios preliminares evidencian que las propuestas iniciales de redes WDM dinámicas experimentan un mayor consumo energético en comparación a las actuales redes WDM estáticas [1516]. Por esta razón es que se hace necesario contar con estrategias de eficiencia energética aplicada a redes ópticas de operación dinámica de la capa WDM.

Las propuestas de estrategias de eficiencia energética para la capa óptica WDM de redes de transporte se han centrado principalmente en redes de operación estática y un número menor en redes WDM de operación dinámica. En [15] solo se evalúa el consumo energético de redes WDM dinámicas y se compara con el de las redes WDM estáticas. Sin embargo, los autores no presentan estrategias de eficiencia energética aplicada a redes WDM dinámicas. En [17], el autor propone un algoritmo de asignación de rutas y longitudes de onda del tipo adaptativo para redes WDM dinámicas, donde su función de peso considera la utilización y el consumo energético. Sin embargo, el autor supone un número arbitrario de longitudes de onda por enlace sin basarse en algún proceso de dimensionamiento que asegure que las conexiones no superen una probabilidad de bloqueo umbral. En [18] se propone un algoritmo de ruteo que representa un compromiso entre el consumo energético y la probabilidad de bloqueo de las nuevas conexiones, sin embargo, el algoritmo está diseñado para redes sin regeneración en los nodos (redes WDM transparentes), el que es un modelo no aplicable en la práctica para redes que cubren grandes extensiones geográficas.

De esta forma, no se han elaborado esquemas de eficiencia energética aplicados a la capa óptica de redes WDM dinámicas que consideren que las conexiones no superen una probabilidad de bloqueo umbral y que los nodos cuenten con regeneradores para reconstruir las señales ópticas. Por esta razón, en este trabajo se propone un mecanismo de funcionamiento de la capa óptica de redes WDM dinámicas donde se controle el número de dispositivos encendidos y apagados de acuerdo con los diferentes valores de carga de tráfico existente a las distintas horas del día y al valor de probabilidad de bloqueo umbral de las peticiones de conexión.

Este artículo se organiza de la siguiente manera: en la sección siguiente se describen los modelos de red, de tráfico, arquitecturas de nodos y enlaces, y el modelo de consumo energético; luego se plantea la propuesta de estrategia de eficiencia energética para redes WDM dinámicas; posteriormente se discuten los resultados numéricos obtenidos, y finalmente se presentan las conclusiones.

\section{MODELOS DE RED, DE TRÁFICO, CONSUMO ENERGÉTICO, ARQUITECTURA DE NODOS Y ENLACES}

Modelo de red: En este artículo se representan las redes ópticas WDM mediante un grafo $G=(N, C)$, 
donde $N$ es el conjunto de nodos ópticos y $C$ representa el conjunto de cables que unen los nodos. El número total de nodos y cables es denotado por $|N|$ y $|C|$, respectivamente. Cada nodo óptico $n \in N$, está localmente conectado a un dispositivo electrónico por una interfaz electroóptica y cada cable $c \in C$ está compuesto por dos fibras ópticas activas o enlaces unidireccionales, denotadas cada una por $l$. El conjunto de fibras ópticas activas en la red es denotado como $L$ y el número total de estas es $|L|=2 \cdot|C|$. En la Figura 1 se muestra una red WDM (representada por la capa WDM) conectada a dispositivos electrónicos (representada por la capa electrónica).

La capacidad requerida por la fibra óptica o enlace unidireccional $l \in L$ (en número de longitudes de onda) está representado por $W_{l}(\rho)$, donde $\rho$ se refiere a la carga de tráfico que ofrece cada par de nodos a la red suponiendo un tráfico ON-OFF. La capacidad total de la red está dada por:

$$
W_{\text {red }}(\rho)=\sum_{\forall l} W_{l}(\rho)
$$

El número de transmisores $(T x)$ y receptores $(R x)$ monocanal (que operan a una longitud de onda $\lambda_{0}$ en particular) requeridos en el nodo para una carga de tráfico ofrecida por cada conexión, $\rho$, son denotados como $|T x(\rho)|_{n} \mathrm{y}|R x(\rho)|_{n}$, respectivamente. El número total de estos dispositivos en la red está dado por: $\Sigma_{\forall n}\left[|T x(\rho)|_{n}+|R x(\rho)|_{n}\right]$.

Para el caso de redes WDM dinámicas, el número de longitudes de onda de cada enlace y número de transmisores y receptores monocanal de cada nodo se determinan mediante un proceso de

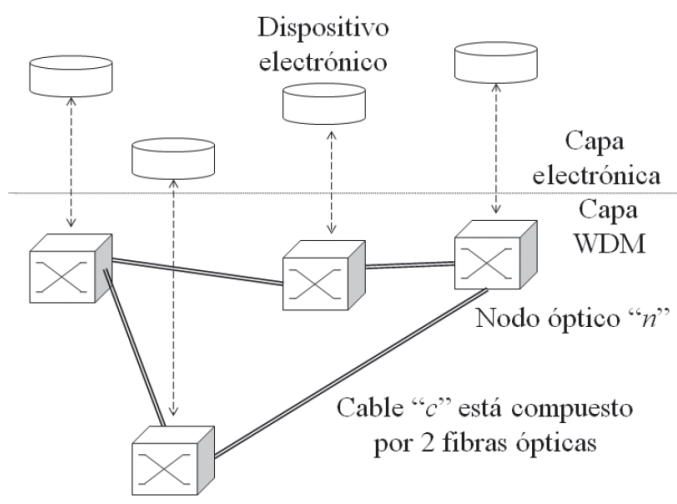

Figura 1. Esquema de una red óptica WDM. dimensionamiento que toma en cuenta la carga de tráfico ofrecida por las conexiones $\rho$ y la probabilidad de bloqueo umbral $P B_{t h}$ [15]. Este proceso de obtención del requerimiento del número de longitudes de onda y transmisores/receptores monocanal puede realizarse de manera secuencial o de manera conjunta. En este artículo se utiliza la manera secuencial por su simplicidad.

Para el establecimiento de conexiones se supone que la red opera con un algoritmo de asignación de rutas y longitudes de onda (RWA: Routing and Wavelength Assignment) con enrutamiento del tipo balanceado, de tal forma que todos los enlaces experimenten el mismo grado de utilización en sus longitudes de onda [19].

Arquitectura de nodos ópticos WDM: En la Figura 2 se muestra la estructura genérica de un nodo óptico (capa WDM), el que se encuentra conectado a un router o dispositivo de transmisión (capa electrónica). El nodo óptico se compone de 4 etapas: de entrada, de conmutación, de interfaz electroóptica y de salida. En la etapa de entrada, la señal óptica WDM que ingresa al nodo desde los enlaces de la red WDM es demultiplexada para posteriormente ser recibida por transpondedores de regeneración o simplemente regeneradores (Reg) con o sin la capacidad de conversión de longitud de onda. Luego, estas señales demultiplexadas son conducidas al dispositivo de conmutación para que sean dirigidas al puerto de salida correspondiente: interfaz electroóptica o etapa de salida, dependiendo si son señales que terminan en el nodo o si son señales en tránsito hacia otros nodos de la red WDM, respectivamente. La interfaz electroóptica, está constituida por transmisores (TTx) y receptores (TRx) WDM, estos son los encargados del intercambio de información entre el nodo en particular (desde o hacia la capa electrónica) y el resto de la red WDM. Los transmisores WDM y receptores WDM cambian la longitud de onda monocanal (denotada como $\lambda_{0}$ en la Figura 2) a una dada por la grilla WDM y viceversa, respectivamente. Debido a que, de acuerdo con la Figura 2, cada transmisor monocanal (Tx) está conectado a un transmisor WDM (TTx) y cada receptor monocal $(\mathrm{Rx})$ está conectado a un receptor WDM (TRx), el número de estos es coincidente en la red. Finalmente, la etapa de salida está constituida por regeneradores (Reg), los que se encargan de acondicionar las señales ópticas de salida para 
luego multiplexarlas para que se transmitan por la fibra óptica correspondiente.

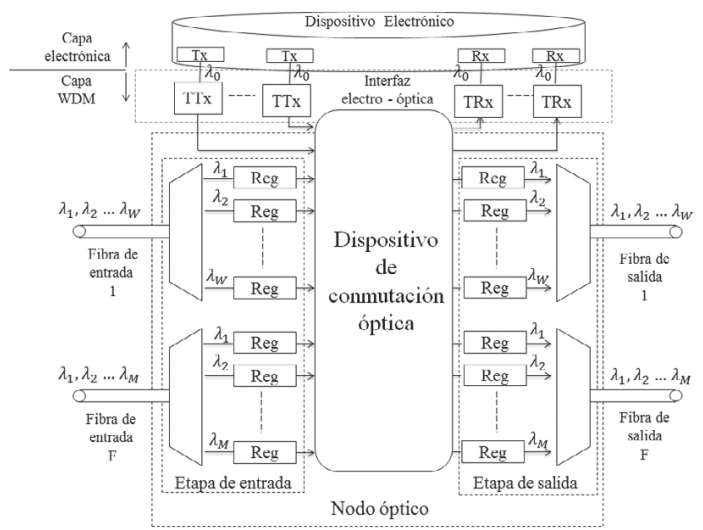

Figura 2. Nodo óptico genérico.

Arquitecturas de nodos ópticos con doble regeneración (en las etapas de entrada y salida) se justifican cuando se tienen dispositivos de conmutación que le producen mucha atenuación y/o distorsión a las señales ópticas [20]. Esto, en conjunto con que estas señales se han propagado por grandes distancias hasta llegar al nodo.

Arquitectura de enlaces WDM: Los enlaces de redes de transporte se extienden por cientos de kilómetros, por lo que requieren de regeneración debido a efectos lineales y no lineales en la fibra que degradan la señal óptica [21]. Típicamente un enlace óptico está constituido por tramos de fibra óptica (FO) y amplificadores ópticos que son los encargados de la compensación de las pérdidas de transmisión en cada tramo. Los amplificadores ópticos más utilizados son los llamados EDFA (Erbium-doped fiber amplifiers). La Figura 3 muestra un esquema general de un enlace de fibra óptica utilizando amplificadores ópticos, los que típicamente se encuentran separados por distancias de 80 a $120 \mathrm{~km}$ [16].

Modelo de tráfico: Cada conexión corresponde a un par de nodos fuente-destino en particular. Se supone que cada conexión genera un mismo tráfico de acuerdo con un proceso ON-OFF [7]. Es decir, durante el periodo $\mathrm{ON}$, el nodo fuente transmite a la máxima velocidad de transmisión digital de la portadora. En cambio, durante el periodo OFF, el

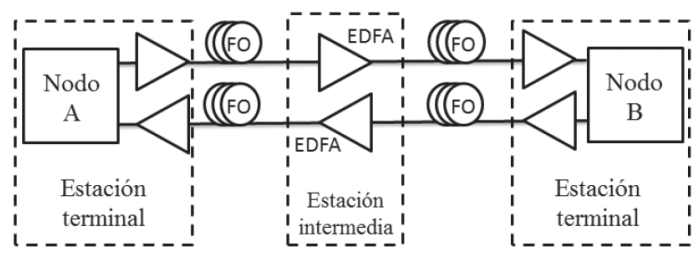

Figura 3. Esquema genérico de un sistema de transmisión de fibra óptica WDM.

nodo fuente se abstiene de transmitir información. La duración media del periodo ON (OFF) es denotada por $t_{O N}\left(t_{O F F}\right)$. De esta forma, la carga de tráfico ofrecida por cada conexión a la red, $\rho$, está dada por:

$$
\rho=\frac{t_{O N}}{t_{O N}+t_{O F F}}
$$

En este artículo se supone un tráfico uniforme, homogéneo y variante en el tiempo. Es decir, que todas las peticiones de conexión tienen la misma probabilidad de ocurrir (uniforme) y que ofrecen la misma carga de tráfico $\rho$ (homogéneo). La característica de variante en el tiempo implica que $\rho$ cambia en cada periodo de observación.

Modelo de consumo de energía de la capa óptica de la red WDM: El consumo energético total de la capa óptica WDM durante un período de observación $T_{j}$, denotado como $E_{\text {red }}\left(T_{j}\right)$ puede ser expresado como:

$$
E_{\text {red }}\left(T_{j}\right)=E_{N}\left(T_{j}\right)+E_{L}\left(T_{j}\right)
$$

Donde $E_{N}\left(T_{j}\right)$ y $E_{L}\left(T_{j}\right)$ representan el consumo total de energía de todos los nodos WDM y de los enlaces de la red medida en $\mathrm{kW}$-h durante un período $T_{j}$, respectivamente. El consumo energético de los nodos se debe a la contribución de mutiplexores/ demultiplexores WDM, regeneradores, transmisores/ receptores WDM y dispositivos de conmutación. Para el caso de los enlaces WDM, la contribución está dada por los amplificadores ópticos. Para obtener el consumo energético de un tipo de dispositivo de la red en un periodo $T_{j}$ se debe multiplicar el consumo de potencia medio de estos individualmente (medido en $\mathrm{kW}$ ) por el tiempo que dura $T_{j}$ y por el número total de estos dispositivos encendidos. 


\section{PROPUESTA DE FUNCIONAMIENTO DE APAGADO Y ENCENDIDO DE DISPOSITIVOS EN UNA RED WDM DINÁMICA}

En este artículo se propone un mecanismo de funcionamiento denominado Load-Aware Turn On-Off, para controlar el número de regeneradores y transmisores/receptores WDM encendidos/ apagados en una red WDM dinámica basados en las variaciones de carga de tráfico $\rho$ de las distintas horas de un día con la finalidad de obtener ahorros energéticos. Todo esto, con la restricción de que no se supere la probabilidad de bloqueo umbral de la red (parámetro de calidad de servicio generalmente utilizado para redes WDM dinámicas [7]). La Figura 4 muestra un patrón de carga de tráfico típico en una conexión de datos, donde se evidencian las grandes variaciones de la carga de tráfico hora a hora [22-23]. En esta se puede apreciar que $\rho_{\max }$ y $\rho_{\text {min }}$ representan los valores de carga de tráfico máxima y mínima experimentada, respectivamente. Valores prácticos para la relación $100 \% \rho_{\min } / \rho_{\max }$ se encuentran en el rango de 5 al 35\% [22].

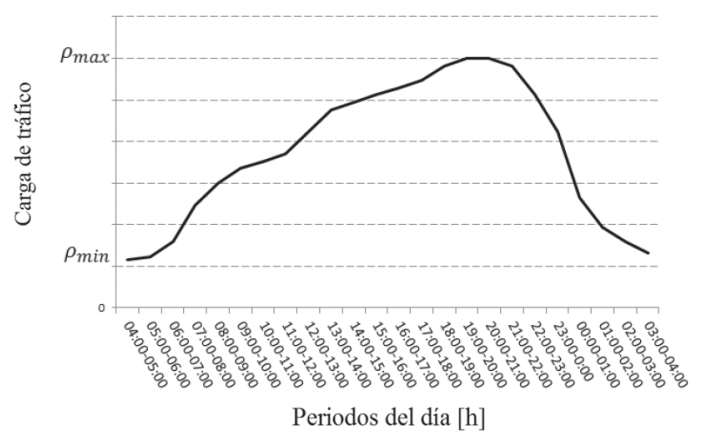

Figura 4. Patrón de carga de tráfico multihora.

Para determinar el número de regeneradores encendidos/apagados se toma en cuenta que este parámetro depende de la capacidad o número de longitudes de onda necesaria en una red [15]. Esta capacidad necesaria en una red WDM dinámica es obtenida con la finalidad de hacer cumplir que las conexiones no superen una probabilidad de bloqueo umbral ante cualquier carga de tráfico. Por esta razón, el número de regeneradores encendidos y/o apagados es dependiente de la carga de tráfico de las conexiones. Por ejemplo, a mayor carga de tráfico, mayor será el número de regeneradores encendidos necesarios para que las conexiones no superen la probabilidad de bloqueo umbral [15].
La Figura 5 muestra un patrón típico de requerimientos de longitudes de onda de una red WDM dinámica (barra achurada) para mantener una probabilidad de bloqueo de las conexiones, el que aumenta a medida que se incrementa la carga de tráfico [15]. En esta se simuló, para cada valor de carga de tráfico ofrecida a la red por las conexiones, el funcionamiento de la topología de la NSFNet (National Science Foundation's Network) como una red WDM dinámica (esto es, procesos de conexión y desconexión considerando una capacidad fija representada por un número de longitudes de onda por cada enlace de la red y un algoritmo RWA con enrutamiento balanceado), para así estimar la probabilidad de bloqueo de las conexiones. Se repite este proceso variando el número de longitudes de onda de la red hasta encontrar el mínimo valor de este parámetro con el que se obtenga la probabilidad de bloqueo umbral en todas las conexiones de la red (criterio de diseño). Este proceso de dimensionamiento se obtuvo suponiendo que existían $N \cdot(N-1)$ transmisores WDM y el mismo número de receptores WDM en la red (un transmisor y un receptor WDM por cada posible destino en la red). De esta forma, considerando el nodo genérico de la Figura 2, el número total de regeneradores necesarios de las etapas de entrada o de salida de una red WDM dinámica (para una carga de tráfico específica $\rho$ y probabilidad de bloqueo umbral $P B_{t h}$ ) está dado por $W_{\text {red }}(\rho)=\Sigma_{\forall l} W_{l}(\rho)$. Requerimientos de dispositivos en cargas de tráfico intermedias a las entregadas por la Figura 5 pueden obtenerse mediante algún método de ajuste de curvas que permita una aproximación. Esto, considerando que a una carga de tráfico cero los requerimientos son nulos y para una carga de tráfico igual a 1 los requerimientos se aproximan a los relacionados a una carga de tráfico igual a 0,9 (estado de saturación para redes con conversión de longitud de onda [15]).

El número necesario de transmisores/receptores WDM $\left(|N| \cdot|T x(\rho)|_{n}+|N| \cdot|R x(\rho)|_{n} \quad\right.$ suponiendo tráfico uniforme y RWA balanceado) en una red dinámica también es dependiente de la carga de tráfico y la probabilidad de bloqueo umbral de esta [15]. La Figura 5 muestra un patrón de requerimiento de transmisores y receptores WDM de una red dinámica (barra blanca) [15]. Este requerimiento se obtuvo mediante un proceso de dimensionamiento vía simulación y secuencial con el proceso de obtención del número de longitudes 
de onda necesarias en la red. Una vez obtenido el número de longitudes de onda necesarias por cada enlace se procede a repetir la simulación disminuyendo en una unidad el número de transmisores/receptores WDM hasta obtener el mínimo requerimiento que satisfaga el valor de la probabilidad de bloqueo umbral. Este procedimiento se realiza por cada valor de carga de tráfico ofrecida por las conexiones a la red. Cabe destacar que cuando la carga de tráfico tiende a 1 , el valor de este parámetro tiende a $2|N|(|N|-1)$, o sea, un par de dispositivos de este tipo para cada posible nodo de destino o conexión. Requerimientos a cargas de tráfico intermedias no entregadas por la Figura 5 pueden obtenerse mediante un ajuste de curva, de la misma forma que para el requerimiento del número de longitudes de onda.

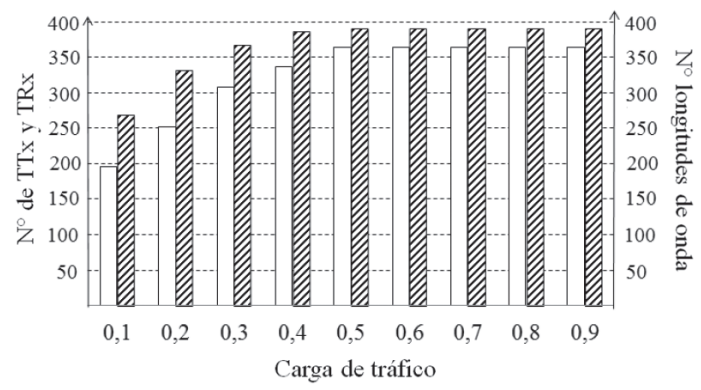

Figura 5. Requerimiento de longitudes de onda (barra achurada) y del número de transmisores y receptores WDM (barra blanca) de la red NSFnet, configurada como una red WDM dinámica, en función de la carga de tráfico para un valor de probabilidad de bloqueo umbral [15].

El funcionamiento del mecanismo Load-Aware Turn On-Off propuesto para redes WDM dinámicas se ilustra en la Figura 6. Para una topología de red con una arquitectura de nodos y enlaces específica, el mecanismo consiste en los siguientes pasos:

Paso 1: Definir la probabilidad de bloqueo umbral o máxima permitida $\left(P B_{t h}\right)$. Este parámetro representa la calidad de servicio (QoS) que el operador de red espera que experimenten las conexiones.

Paso 2: Obtener la curva de requerimientos de longitudes de onda de la red $\left(W_{\text {red }}(\rho)\right)$ para cumplir con una probabilidad de bloqueo dada y para diferentes cargas de tráfico. Esta curva se debe obtener por un método de dimensionamiento para redes WDM dinámicas [7] considerando un algoritmo RWA con enrutamiento balanceado, tráfico uniforme y homogéneo [7, 19, 24-25] (ver ejemplo de la Figura 5 y explicación asociada). De esta forma, el número de longitudes de onda necesarios es el mismo para cada enlace de la red. Con esta información es posible obtener el número de regeneradores $(\mathrm{Reg})$ requeridos en la red. $\mathrm{Si}$ utiliza un nodo como el de la Figura 2, cada longitud de onda de la red tiene asociado un regenerador al inicio y final de esta en una fibra óptica. Para este caso, el número total de regeneradores (en la etapa de entrada más de los de la etapa de salida en todos los nodos de la red) es igual al doble del número de longitudes de onda total de la red, es decir, $2 \cdot W_{\text {red }}(\rho)=2 \cdot \Sigma_{\forall l} W_{l}(\rho)=2 \cdot|L| \cdot W_{l}(\rho)$.

Paso 3: Obtener la curva de requerimientos de transmisores y receptores WDM para cumplir una probabilidad de bloqueo dada y para diferentes cargas de tráfico. Esta curva se debe realizar con un método de dimensionamiento de este tipo de dispositivos [15, 26-27], el que debe considerar la probabilidad de bloqueo umbral de las conexiones, la carga de tráfico (uniforme y homogéneo) y algoritmo RWA con enrutamiento balanceado. Ver ejemplo de la Figura 5 y explicación asociada. De esta forma, el número de estos dispositivos será el mismo para cada nodo de la red.

Paso 4: En cada cambio de hora y para el funcionamiento en un periodo $T_{j}$ se debe realizar lo siguiente:

4.a) Estimar la máxima carga de tráfico ofrecida por las conexiones ( $\rho$ ) del periodo a ocurrir $T_{j}$, la que denominaremos $\rho_{\max }\left(T_{j}\right)$. Esto se realiza considerando el peor caso de carga de tráfico que pudiese ocurrir en el periodo. Para realizar esto se debe utilizar algún mecanismo de predicción de la carga de tráfico. Por ejemplo: se podría elaborar, por medio de mediciones, una curva de la carga de tráfico histórica en función de las horas del día (como en la Figura 4), la que sea representativa para las conexiones de la red. Esta podría registrar los máximos históricos. Entonces, en cada cambio de hora se selecciona el valor de carga de tráfico máxima que se ha experimentado históricamente en el periodo a ocurrir $\rho_{\max }\left(T_{j}\right)$.

4.b) Obtener el número necesario de regeneradores (Reg) encendidos en las etapas de entrada y 
salida de los nodos de la red en el período $T_{j}$. Este número debe obtenerse de acuerdo con los resultados del Paso 2 para una carga de tráfico $\rho_{\max }\left(T_{j}\right)$ (Paso 4.a) y probabilidad de bloqueo umbral dadas (Paso 1). De esta forma, el número total de regeneradores de la red que debiesen estar encendidos en el periodo $T_{j}$ corresponde a $2 \cdot W_{\text {net }}\left(\rho_{\max }\left(T_{j}\right)\right)$.

4.c) Obtener el número necesario de transmisores WDM (TTx) y receptores WDM (TRx) encendidos de la etapa de interfaz electroóptica de los nodos de la red en el período $T_{j}$. Este número debe obtenerse de acuerdo con los resultados del Paso 3 para la carga de tráfico $\rho_{\max }\left(T_{j}\right)$ del Paso 4.a y la probabilidad de bloqueo umbral del Paso 1 en el periodo en estudio. El número total de transmisores WDM que deben estar encendidos en la red durante el período $T_{j}$ está dado por: $|N| \cdot \mid T x\left(\rho_{\max }\right.$ $\left.\left(T_{j}\right)\right)\left.\right|_{n}$. Este valor es equivalente al número de receptores WDM que debiesen estar encendidos durante el período $T_{j}$.

4.d) Encender o apagar el número de dispositivos necesarios (Reg, TTx y TRx) de los nodos de la red para alcanzar los resultados de los Pasos 4.b y 4.c. Este procedimiento se debe realizar de manera homogénea (automáticamente en la capa de control de la red), es decir, el número de los dispositivos a encender o a apagar en los nodos debe ser igual en cada uno de ellos. Este número se debe obtener de acuerdo con los resultados de los Pasos 4.b (número de regeneradores encendidos) y 4.c (número de transmisores y receptores WDM encendidos) para el periodo en estudio y restarlos con los resultados del periodo anterior, para cada caso. Si el valor es positivo, entonces se debe encender ese número de dispositivos. En caso contrario, se deben apagar. Así, el número de dispositivos regeneradores que se debiesen encender o apagar en cada nodo para el período $T_{j}$ está dado por: $2 \cdot\left[W_{\text {net }}\left(\rho_{\max }\left(T_{j}\right)\right)-\right.$ $\left.W_{\text {net }}\left(\rho_{\max }\left(T_{j-1}\right)\right)\right]$. Para el caso del número de transmisores WDM que se debiesen encender o apagar en la red para el período $T_{j}$, este está dado por la siguiente expresión: [ $\mid \operatorname{Tx}\left(\rho_{\max }\right.$ $\left.\left.\left(T_{j}\right)\right)\left.\right|_{n}-\left|\operatorname{Tx}\left(\rho_{\max }\left(T_{j-1}\right)\right)\right|_{n}\right]$. Para el caso de los receptores WDM, este número es coincidente con el de los transmisores WDM.

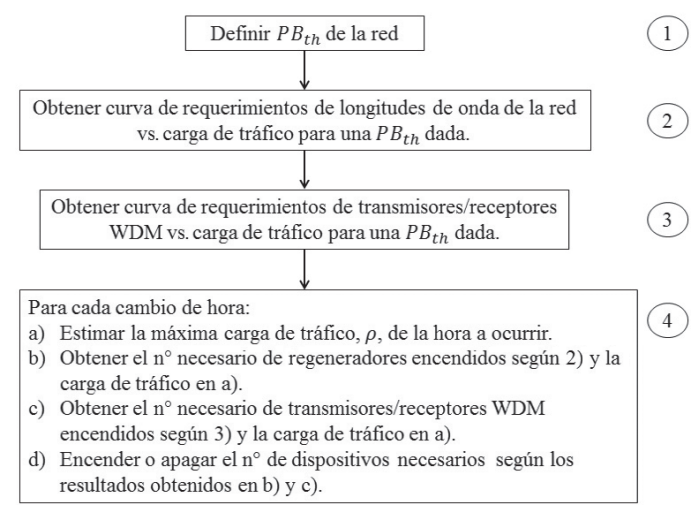

Figura 6. Mecanismo propuesto Load-Aware Turn On-Off.

\section{RESULTADOS NUMÉRICOS}

El mecanismo de eficiencia energética Load-aware Turn On-Off fue evaluado para la topología NSFNet (14 nodos y 21 cables o 42 fibras ópticas activas) configurada como red WDM dinámica operando con conexiones con una tasa de transmisión digital de 40 Gbps. La Figura 7 muestra la topología considerada.

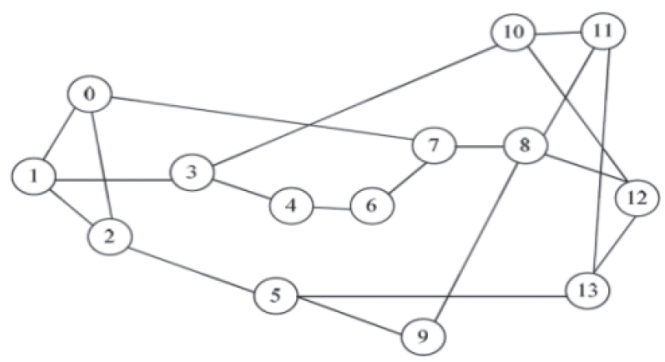

Figura 7. Topología NSFNet.

El nodo WDM dinámico considerado en este artículo se muestra en la Figura 8 [15]. Se puede apreciar que:

- La etapa de entrada se compone de demultiplexores WDM y de regeneradores sintonizables LR-SR (long-reach y short-reach). Los regeneradores de esta etapa, como función adicional a la regeneración, convierten la portadora de cada señal a una longitud de onda necesaria de acuerdo con los requerimientos del dispositivo de conmutación.

- La etapa de conmutación se compone de un AWG (Arrayed Waveguide Grating), el que tiene una matriz de conmutación fija para cada 
longitud de onda. Es decir, el AWG tiene tantas matrices de conmutación fijas como longitudes de onda tiene el sistema. De esta forma, para escoger un puerto de salida del AWG se debe sintonizar la longitud de onda adecuada en un puerto de entrada de este dispositivo de conmutación [15].

- La etapa de interfaz electroóptica se compone de transmisores WDM sintonizables SR-SR y receptores WDM no sintonizables SR-SR [15].

- La etapa de salida se compone de regeneradores no sintonizables SR-LR. Estos dispositivos optoelectrónicos son los encargados de la regeneración y conversión de longitud de onda de la señal óptica de salida.

En este análisis se excluye el consumo de los amplificadores ópticos debido a que ambas opciones (con y sin estrategia de eficiencia energética) requieren el mismo número de estos dispositivos.

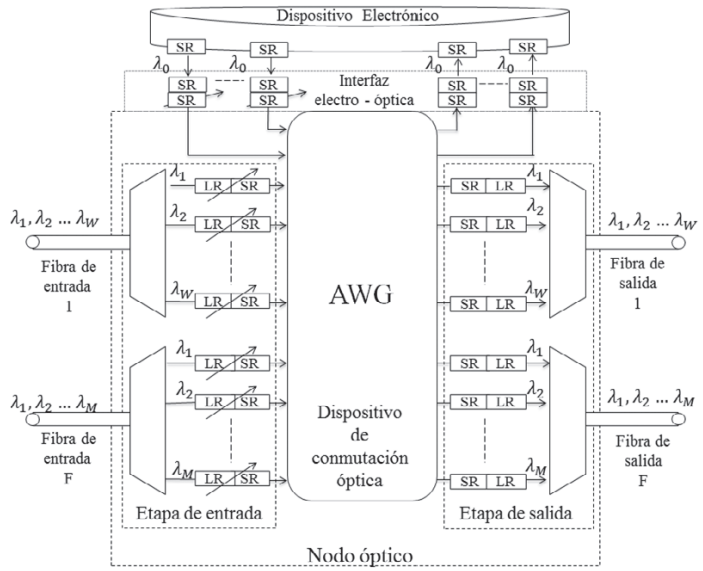

Figura 8. Nodo WDM dinámico [15, 20].

Los valores de consumo de potencia promedio de los dispositivos considerados en este artículo se muestran en la Tabla 1.

La aplicación del mecanismo Load-Aware Turn On-Off se muestra a continuación:

Paso 1: Se define el valor $P B_{t h}=10^{-3}$, el que ha sido frecuentemente usado en la literatura [7, 15, 29].

Paso 2: Los valores de los requerimientos de regeneradores de las etapas de entrada más los de la etapa de salida $\left(2 \cdot W_{\text {red }}(\rho)\right)$ para la NSFNet se muestran en la Tabla 2 [15]. Estos valores fueron obtenidos para una probabilidad de bloqueo umbral
Tabla 1. Valores en término de potencia nominal de los dispositivos de red considerados [28].

\begin{tabular}{|l|c|}
\hline \multicolumn{1}{|c|}{ Nombre y descripción } & [W] \\
\hline $\begin{array}{l}\text { TTx: Transmisor WDM SR-SR sintonizable de } \\
\text { 40 Gbps (etapa de interfaz electroóptica). }\end{array}$ & 15 \\
\hline $\begin{array}{l}\text { TRx: Receptor WDM SR-SR no sintonizable de } \\
\text { 40 Gbps (etapa de interfaz electroóptica). }\end{array}$ & 15 \\
\hline Demultiplexor / Multiplexor WDM & 0 \\
\hline $\begin{array}{l}\text { Reg: Regenerador LR-SR sintonizable de 40 } \\
\text { Gbps. de 1.500 km de alcance (etapa de entrada) }\end{array}$ & 70 \\
\hline $\begin{array}{l}\text { Reg: Regenerador SR-LR no sintonizable de 40 } \\
\text { Gbps. de 1.500 km de alcance (etapa de salida) }\end{array}$ & 70 \\
\hline Dispositivo de conmutación AWG. & 0 \\
\hline
\end{tabular}

de $10^{-3}$ y usando algoritmos de enrutamiento fijo [19] tal, que las rutas escogidas produjeran una utilización balanceada de los enlaces (la tabla de ruteo se diseña para que cada enlace tenga asignada la misma cantidad de rutas).

Paso 3: Los valores de los requerimientos de transmisores más receptores WDM $\left(2 \cdot|N| \cdot|T x(\rho)|_{n}\right)$ para la NSFNet se muestran en la Tabla 2 [15]. Estos valores fueron obtenidos bajo las mismas condiciones del requerimiento del número de regeneradores.

Paso 4:

4.a) Con fines didácticos, para obtener $\rho_{\max }\left(T_{j}\right)$ el tráfico multihora considerado en este artículo es similar al de la Figura 4, donde $\rho_{\max }$ y $\rho_{\min }$ se suponen igual a 0,3 y 0,06 , respectivamente. De esta forma, la relación $100 \% \cdot \rho_{\min } / \rho_{\max }$ es igual a $20 \%$, el que se encuentra en el rango típico reportado para este parámetro $(5 \mathrm{al} 35 \%$ $[22,30])$. Para obtener los valores intermedios de la carga de tráfico se realiza un ajuste de curva de acuerdo con el patrón de la Figura 4. 4.b) Una vez obtenidos los valores de los requerimientos de regeneradores de entrada y de salida (ver Tabla 2) para distintas cargas de tráfico de 0,1 a 0,9 , se procede a determinar el número de estos dispositivos que deben estar encendidos para las distintas horas del día de acuerdo con el tráfico multihora de la red considerada. Los resultados se muestran en la Figura 9.

4.c) Para determinar el número de transmisores y receptores WDM que deben estar encendidos a cada hora se toman en cuenta los valores de este requerimiento de la Tabla 2 para así relacionarlos 
con el valor de la carga de tráfico máxima de cada hora. Los resultados se muestran en la Figura 9.

4.d) El número de dispositivos necesarios a encender (valores positivos) o a apagar (valores negativos) para cada periodo se muestran en la Figura 10. En esta, el número de transmisores y receptores WDM a encender o apagar se representa en la porción blanca de las barras, en cambio para el caso de los regeneradores, con la barra completa.

Tabla 2. Número de regeneradores y de transmisores/ receptores WDM necesarios para la NSFNet [15].

\begin{tabular}{|c|c|c|c|c|c|c|}
\cline { 2 - 7 } \multicolumn{1}{c|}{} & \multicolumn{6}{c|}{ Carga de tráfico ofrecida a la red } \\
\cline { 2 - 7 } \multicolumn{1}{c|}{} & 0,1 & 0,2 & 0,3 & 0,4 & 0,5 & $0,6-0,9$ \\
\hline $2 \cdot W_{\text {red }}(\rho)$ & 538 & 662 & 734 & 772 & 780 & 780 \\
\hline $2 \cdot|N| \cdot|T x(\rho)|_{n}$ & 196 & 252 & 308 & 336 & 364 & 364 \\
\hline
\end{tabular}

Considerando los valores de consumo energético de cada componente, de acuerdo con la Tabla 1, y a los requerimientos del número de dispositivos para el tráfico multihora considerado para la NSFNet (Figura 9), se construyó una gráfica del consumo energético total de la red para cada hora del día (Figura 11). Además se incluye el consumo energético para una red WDM dinámica diseñada sin técnicas de eficiencia energética, esto es, para satisfacer la carga de tráfico máxima considerada, esta corresponde en este ejemplo a $0,32 \cdot W_{\text {red }}(\rho)$ igual a 734 y $2 \cdot|N| \cdot|T x(\rho)|_{n}$ igual a 308$)$.

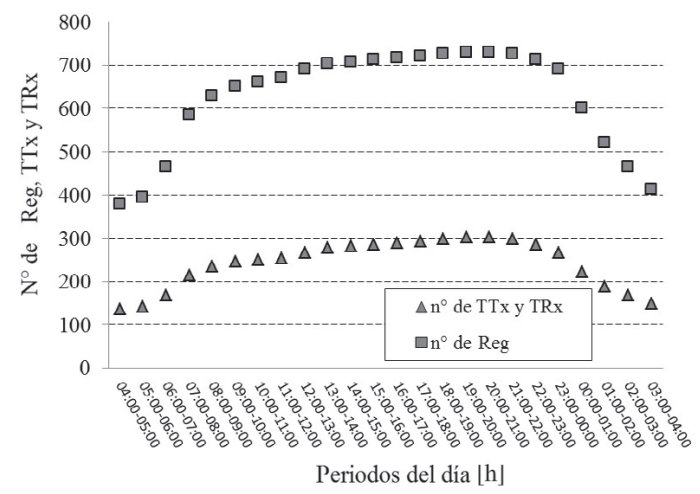

Figura 9. Requerimientos de regeneradores (cuadrado) y transmisores más receptores WDM (triángulo) encendidos en función de las distintas horas del día para la NSFNet [15].

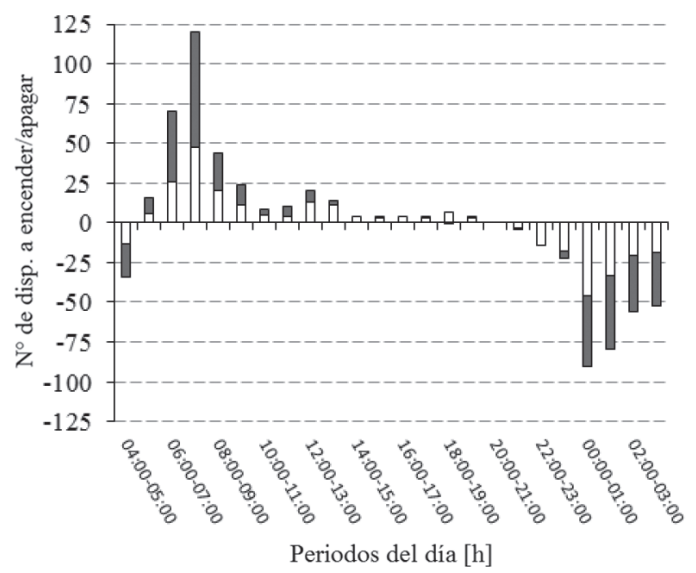

Figura 10. Número de regeneradores (barra completa) y de transmisores/receptores WDM (sección blanca de las barras) a apagar/ encender en cada hora del día.

En la Figura 11 se puede apreciar claramente que la propuesta de eficiencia energética alcanza un ahorro energético en los nodos en las horas de menor carga de tráfico en comparación a la opción dinámica sin esta estrategia.

Además, en un día se alcanzan ahorros de 200 kW-h, lo que implica un ahorro del 15\% en comparación al caso dinámica sin estrategia de eficiencia energética. Se alcanzan en algunas horas específicas ahorros de $25 \mathrm{kWh}$ cuando las conexiones le ofrecen a la red la mínima carga de tráfico. El mecanismo logra estos

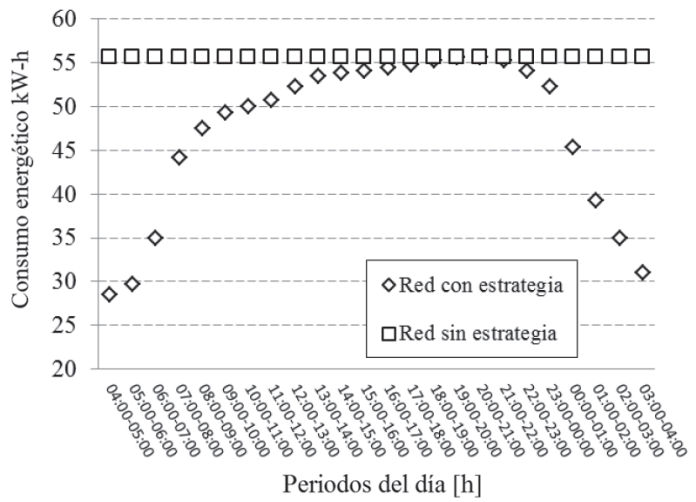

Figura 11. Consumo energético hora a hora de los transmisores/receptores WDM y regeneradores de la NSFNet configurada como red WDM dinámica con (rombo) y $\sin$ (cuadrado) estrategia de eficiencia energética. 
ahorros energéticos asegurando una probabilidad de bloqueo umbral para las conexiones.

\section{CONCLUSIONES}

En este artículo se propuso un mecanismo de funcionamiento de redes WDM dinámicas denominado Load-Aware Turn On-Off. Este está basado en controlar el encendido y apagado de regeneradores y transmisores/receptores WDM de los nodos en función de la carga multihora con la finalidad de lograr ahorros en el consumo energético y asegurar una probabilidad de bloqueo umbral de las conexiones.

Se evaluó el consumo energético de esta propuesta para la topología física NSFNet como red WDM dinámica con nodos basados en AWG y se comparó con una sin esta estrategia. Resultados muestran que esta propuesta de eficiencia energética logra ahorros de $15 \%$.

Estos resultados pueden ser de interés a los operadores de telecomunicaciones que se encuentren evaluando la factibilidad de implementar redes WDM dinámicas en un futuro.

Trabajos futuros debieran centrarse en realizar las modificaciones de esta metodología para que sea aplicable en condiciones de tráfico distintas a las supuestas en este trabajo (no uniforme y heterogéneo), un ruteo que no permita un balance de carga en los enlaces y un dimensionamiento conjunto de dispositivos. Con esto, se podría permitir un encendido/apagado de dispositivos de manera no uniforme y alcanzar mayores ahorros energéticos.

\section{AGRADECIMIENTOS}

A los proyectos DII-PUCV 037.327/2013, 037.325/2013 y Fondecyt de Iniciación 11121437 por el apoyo financiero y al "Programa de Ayudantes de Investigación" de la Escuela de Ingeniería Eléctrica de la PUCV.

\section{REFERENCIAS}

[1] L. Chiaraviglio, M. Mellia and F. Neri. "Reducing Power Consumption in Backbone Networks". IEEE International Conference on
Communications (ICC). Dresden, Germany. June, 2009.

[2] A. Saleh and J. Simmons. "Technology and Architecture to Enable the Explosive Growth of the Internet". IEEE Communications Magazine. Vol. 49, Issue 1, pp. 126-132. January, 2011. ISSN: 0163-6804. DOI: 10.1109/MCOM. 2011.5681026.

[3] R. Tucker. "Green Optical CommunicationPart I: Energy Limitations in Transport". IEEE Journal of selected topics in quantum electronics. Vol. 17, Issue 2, pp. 245-260. April, 2011. ISSN: 1077-260X. DOI: 10.1109/ JSTQE.2010.2051216.

[4] G. Koutitasa and P. Demestichas. "A review of energy efficiency in telecommunication Networks". Telfor Journal. Vol. 2, Issue 1, pp. 2-7. 2010. ISSN: 1821-3251.

[5] Y.Zhang, P. Chowdhury, M. Tornatore and B. Mukherjee. "Energy Efficiency in Telecom Optical Networks". IEEE Communications Surveys \& Tutorials. Vol. 2, Issue 4, pp. 441458. July, 2010. ISSN: 1553-877X. DOI: 10.1109/SURV.2011. 062410.00034.

[6] A. Sano, T. Kobayashi, S. Yamanaka, A. Matsuura, H. Kawakami, Y. Miyamoto, K. Ishihara and H. Masuda. "102.3-Tb/s $(224 \times 548-\mathrm{Gb} / \mathrm{s}) \mathrm{C}$ - and extended L-band all-Raman transmission over $240 \mathrm{~km}$ using PDM-64QAM single carrier FDM with digital pilot tone". Optical Fiber Communication Conference and Exposition (OFC/NFOEC). Los Angeles, USA. March, 2012.

[7] A. Zapata-Beghelli and P. Bayvel. "Dynamic versus static wavelength-routed optical networks". Journal of Lightwave Technology. Vol. 26, Issue 20, pp. 3403-3415. October, 2008. ISSN: 0733-8724. DOI: 10.1109/ JLT.2008.925718.

[8] D. Hunter and I. Andonovic. "Approaches to Optical Internet Packet Switching". IEEE Communications Magazine. Vol. 38, Issue 9, pp. 116-122. September, 2000. ISSN: 01636804. DOI: $10.1109 / 35.868150$.

[9] C. Qiao and M. Yoo. "Optical burst switching (OBS) - a new paradigm for an Optical Internet”. Journal of High Speed Networks. Vol. 8, Issue 1, pp. 69-84. March, 1999. ISSN: $1875-8940$.

[10] M. Düser and P. Bayvel. "Analysis of a dynamically wavelength-routed optical burst 
switched network architecture". Journal of Lightwave Technology. Vol. 20, Issue 4, pp. 574-585. April, 2002. ISSN: 0733-8724. DOI: $10.1109 / 50.996576$.

[11] A. Jajszczyk. "Automatically Switched Optical Networks: Benefits and Requirements". IEEE Communications Magazine. Vol. 43, Issue 2, pp. S10-S15. February, 2005. ISSN: 01636804. DOI: 10.1109/MCOM.2005.1391497.

[12] A. Patel, P. Ji, J. Jue and T. Wang. "Dynamic routing, wavelength assignment, and spectrum allocation in transparent flexible optical WDM networks". Proc. SPIE 7959. Optical Metro Networks and Short-Haul Systems III. 79590M. January 24, 2011. DOI: 10.1117/12.876407.

[13] H.-C. Leligou. "Efficient burst aggregation for QoS-aware slotted OBS systems". European Transactions on Telecommunications. Vol. 17, Issue 1, pp. 93-98. January, 2006. ISSN: 1541-8251. DOI: 10.1002/ett.1096.

[14] M. Düser. "Investigation of the impact of traffic growth and variability on future network architectures". European Conference on Optical Communications (ECOC). Glasglow, Scotland. September, 2005.

[15] A. Leiva, J.M. Finochietto, B. Huiszoon, V. López, M. Tarifeño, J. Aracil and A. Beghelli. "Comparison in Power Consumption of Static and Dynamic WDM Networks". Optical Switching and Networking. Special Issue on Green Communications and Networking. Vol. 8, Issue 3, pp. 149161. July, 2011. ISSN: 1573-4277. DOI: 10.1016/j.osn.2011.03.005.

[16] A. Leiva, C. Mas and A. Beghelli. "Upgrading cost modelling of capacity-exhausted static WDM networks". Conference on Optical Network Design and Modeling (ONDM). Colchester, UK. April, 2012.

[17] C. Cavdar. "Energy-efficient Connection Provisioning in WDM Optical Networks". Optical Fiber Communication Conference and Exhibit (OFC/NFOEC). Paper OWI3. Los Angeles, USA. March, 2011.

[18] P. Wiatr, P. Monti and L. Wosinska. "Power Savings versus Network Performance in Dynamically Provisioned WDM Networks". IEEE Communications Magazine. Vol. 50, Issue 5, pp. 48-55. May, 2012. ISSN: 01636804. DOI: 10.1109/MCOM.2012.6194382.
[19] H. Zang, J.P. Jue and B. Mukherjee. "A review of routing and wavelength assignment approaches for wavelength-routed optical WDM networks". Optical Networks Magazine. Vol. 1, Issue 1, pp. 47-60. January, 2000. ISSN: 1388-6916.

[20] Y. Huang, D. Datta, X. Qiu, J. Zhang, H.K. Park, Y.C. Kim, J.P. Heritage and B. Mukherjee. "Studies on a Class of AWGBased Node Architectures for Optical Burst-Switched Networks". In Proc. of International Conference on Computational Science (ICCS 2004). Vol. 3038, pp. 12241232. 2004. ISSN: 978-3-540-24688-6. DOI: 10.1007/978-3-540-24688-6_158.

[21] B. Chomycz. "Planning Fiber Optics Networks". McGraw-Hill. 1st Ed. 2009.

[22] R. Bolla, R. Bruschi, A. Cianfrani and M. Listanti. "Enabling Backbone Networks to Sleep". IEEE Network. Vol. 25, Issue 2, pp. 26-31. April, 2011. ISSN: 0890-8044. DOI: 10.1109/MNET.2011.5730525.

[23] AMS-IX. "Statistics". Fecha de actualización: Mayo 2014. Fecha de consulta: Mayo 2014. URL: http://www.ams-ix.net/statistics

[24] G.P.V. Thodime, V.M. Vokkarane and J.P. Jue. "Dynamic congestion-based load balanced routing in optical burst-switched networks". Global Telecommunications Conference (GLOBECOM). San Francisco, USA. 2003.

[25] A. Mokhtar and M. Azizoğlu. "Adaptive wavelength routing in all-optical networks". IEEE/ACM Transactions on Networking. Vol. 6, Issue 2, pp. 197-206. April, 1998. ISSN: 1063-6692. DOI: 10.1109/90.664268.

[26] M. Tarifeño, A. Beghelli and E. Moreno. "Optimal dimensioning of dynamic WDM networks". Conference on Optical Networking Design and Modeling (ONDM). Bologna, Italy. February, 2011.

[27] O. Turkcu and S. Subramaniam. "Transponder Wavelength Assignment in WDM Networks". Optical Fiber Communication Conference and Exhibit (OFC/NFOEC). Paper OThQ4. San Diego, USA. February, 2008.

[28] A. Leiva, C. Machuca, A. Beghelli and R. Olivares. "Migration cost analysis for upgrading WDM networks". IEEE Communications Magazine. Vol. 51, Issue 11, pp. 87-93. November, 2013. ISSN: 01636804. DOI: 10.1109/MCOM.2013.6658658. 
[29] A. Zapata and P. Bayvel. "Do we really need dynamic wavelength-routed optical networks?'. High Performance Computing and Communications. Lecture Notes in Computer Science. Vol. 4208, pp. 477-486. 2006. ISSN: 0302-9743. DOI: 10.1007/11847366_49.
[30] C. Lange and A. Gladisch. "Energy Efficiency Limits of Load Adaptive Networks". Optical Fiber Communication and National Fiber Optic Engineers Conference (OFC/NFOEC). San Diego, USA. March, 2010. 